\title{
STIR-RST: A Software tool for reactive smart tracer studies
}

DOI:

10.1016/j.envsoft.2020.104894

\section{Document Version}

Accepted author manuscript

Link to publication record in Manchester Research Explorer

\section{Citation for published version (APA):}

Bottacin-busolin, A., Dallan, E., \& Marion, A. (2021). STIR-RST: A Software tool for reactive smart tracer studies. Environmental Modelling \& Software, 135. https://doi.org/10.1016/j.envsoft.2020.104894

\section{Published in:}

Environmental Modelling \& Software

\section{Citing this paper}

Please note that where the full-text provided on Manchester Research Explorer is the Author Accepted Manuscript or Proof version this may differ from the final Published version. If citing, it is advised that you check and use the publisher's definitive version.

\section{General rights}

Copyright and moral rights for the publications made accessible in the Research Explorer are retained by the authors and/or other copyright owners and it is a condition of accessing publications that users recognise and abide by the legal requirements associated with these rights.

\section{Takedown policy}

If you believe that this document breaches copyright please refer to the University of Manchester's Takedown Procedures [http://man.ac.uk/04Y6Bo] or contact uml.scholarlycommunications@manchester.ac.uk providing relevant details, so we can investigate your claim.

\section{OPEN ACCESS}




\title{
STIR-RST: A Software Tool for Reactive Smart Tracer Studies
}

\author{
A. Bottacin-Busolin ${ }^{1}$, E. Dallan ${ }^{2}$ and A. Marion ${ }^{2}$
}

${ }^{1}$ Department of Mechanical, Aerospace and Civil Engineering, University of Manchester, Manchester, M13 9PL, UK

Email: andrea.bottacinbusolin@manchester.ac.uk

${ }^{2}$ Department of Industrial Engineering, University of Padua, via F. Marzolo 9, 35131 Padova, Italy

Email edallan@hotmail.it, andrea.marion@unipd.it

Corresponding author: Andrea Bottacin-Busolin (andrea.bottacinbusolin@manchester.ac.uk)

\begin{abstract}
The introduction of "smart" tracer techniques in recent years has provided new ways to investigate sediment-water interactions and microbial activity in stream corridors. In this study, the formulation of the STIR model (Marion et al., 2008) is extended to represent the transport and transformation of Resazurin-Resorufin smart tracers, and an object-oriented toolbox, STIR-RST, is presented for model evaluation and calibration. STIR-RST allows different storage processes to be represented by specific residence time distributions (RTDs), with two possible arrangements of the storage zones: nested (in-series) or competing (in-parallel). The application of STIR-RST to field tracer data is demonstrated assuming two storage zones with exponential RTD. Results show that the assumption of two storage zones provides a better approximation of the observed BTCs compared to that of a single storage zone, at the cost of higher parameter uncertainty. Similar fits are obtained for nested and competing zone arrangements.
\end{abstract}

\section{Keywords}

solute transport; modelling; smart tracers; Resazurin; Resorufin; multiple storage zones

\section{Software availability}

Program title: STIR-RST

Contact address: andrea.bottacinbusolin@manchester.ac.uk

Software access: www.github.com/andreabottacin/STIR-RST

Year first available: 2020

Software requirements: Matlab R2017a or later

Program language: Matlab 
Availability and cost: open source

License: GPL3

\section{Introduction}

Understanding solute transport processes in rivers is essential for improving water quality in surface waters and providing relevant ecological services. The fate of solutes in streams and rivers is influenced by surface-subsurface interactions and biogeochemical processes. Over the last few decades, these mechanisms have been extensively studied, and models and experimental techniques have been developed to analyze the transport of nutrients and pollutants in stream corridors. Usually, reach-averaged model parameters are calibrated using data from tracer tests. Most solute transport models are based on the advection-dispersion equation with additional mass transfer terms representing exchange and temporary trapping in storage zones. A well-known model is the Transient Storage Model (TSM), presented by Bencala and Walters (1983), which assumes advective exchange with a storage zone of finite size. The TSM is implemented in the widely used software OTIS developed by Runkel and Chapra (1993) and its extended version for parameter calibration OTIS-P (Runkel, 1998). A two-storage zone extension of the TSM, generally referred to as TSZM, was initially proposed by Choi et al. (2000) and later applied in several stream tracers studies (TSZM) (e.g., Gooseff et al., 2004; Briggs et al., 2009; Bottacin-Busolin et al., 2011; Zaramella et al., 2016). In the TSZM, a conceptual separation is made between surface transient storage (STS; e.g., side pockets, vegetated zones) and hyporheic transient storage (HTS).

Alternative 1-D transport models with hyporheic exchange have been proposed by several authors. Haggerty and Reeves (2002) developed the Solute Transport and Multirate Mass Transfer-Linear Coordinates (STAMMT-L), which is based on the advection-dispersion mass transfer equation (ADMTE) with a source-sink term accounting for mass exchange with immobile (storage) domains. Wörman et al. (2002) proposed the Advective Storage Path (ASP) model, which represents hyporheic exchange using a residence time distribution derived from the advective pumping theory (Elliott and Brooks, 1997). Deng et al. (2006) presented an application of the fractional advection-dispersion equation (FADE) to solute transport in rivers. A general parameter estimation software for fractional transport models named FracFit was later presented by Kelly et al. (2017). Boano et al. (2007) presented an application of the Continuous Time Random Walk (CTRW) theory as a general modelling framework to represent solute transport in rivers, and used a power-law residence time distribution to represent bed form induced hyporheic exchange. Marion et al. (2008) proposed a general 
residence time formulation for solute transport in rivers (STIR) in which different storage processes are represented by different residence time distributions (RTDs) and exchange rates. STIR was later shown to well represent field tracer BTCs when two storage zones with exponential RTDs are assumed (Bottacin-Busolin et al., 2011; Zaramella et al., 2016). More recently, Bottacin-Busolin (2019) presented a one-dimensional transport model assuming exponentially attenuated mixing (EAM) in the hyporheic zone and showed that this model has similar performance to the TSZM in representing field tracer BTCs, although the scaling of the moments of the BTCs predicted by the two models as a function of the distance from the injection point is generally inconsistent with each other.

As an alternative to physically based approaches, a model founded on data-based mechanistic (DBM) principles was proposed by Beer and Young (1983) and is commonly referred to as Aggregated Dead Zone (ADZ) model. Later experimental studies have shown that ADZ model can well reproduce the shape of tracer BTCs if the order of the underlying transfer function is sufficiently high (e.g., Wallis et al., 1989; Young and Garnier, 2006; Smith et al., 2006). However, the lack of a direct physical interpretation of the model parameters makes DBM methods less suited for inverse modelling studies that specifically aim to characterise physical stream processes.

In the context of physically based approaches, multiple storage zone models such as those presented by Choi et al. (2000) or Marion et al. (2008) assume that the exchange with the storage zone has a "competing" structure, meaning that the storage zones act in parallel, exchanging mass with the main flow channel (MFC) but not between storage zones. Kerr et al. (2013) proposed a two-storage zone model with a "nested" structure, in which storage zones are arranged in series: the main flow channel exchanges with the surface storage zone, and the surface storage zone exchange with the hyporheic zone. Using a reactive tracer, their study demonstrated that calibrated model parameters are affected by the model structure.

Transient storage zones also differ for their biogeochemical characteristics, and hence microbial activity. Tracer techniques based on conservative tracers provide information about transport parameters and travel times, but they have limitations in distinguishing the different stream compartments. In recent years, the development of "smart" tracer techniques has provided a way to obtain additional information about microbiological activity and sedimentwater interactions. Haggerty et al. (2008) proposed the use of Resazurin (Raz, blue color) as a smart tracer, which is converted to Resorufin (Rru, fluorescent pink color) under reducing conditions. The Raz-Rru system has been used to quantify physical transport parameters and microbial metabolic activity in aquatic ecosystems at multiple scales (Knapp et al., 2018). The 
application of Raz as a tracer has been demonstrated by several reach-scale studies (e.g., Argerich et al., 2011; Lemke, Liao, et al.,2013; González-Pinzón et al., 2014, 2015, 2016). Together with new experimental techniques, relevant models have been developed for representing transport and biochemical processes. For example, Argerich et al. (2011) developed a model separating transient storage in a metabolically active component and a metabolically inactive component (MATS-MITS model). Yakirevich et al. (2017) developed a smart tracer model separating surface and hyporheic transient storage and compared the results with the MATS-MITS model, finding better fits but higher uncertainty if all reaction parameters are calibrated for all storage compartments. Liao and Cirpka (2011) and Liao et al. (2013) proposed a shape-free approach for deriving the hyporheic travel time distribution from conservative and smart tracer experiments. This approach was later improved by Knapp and Cirpka (2017) who developed a local-in-global optimization approach for the inference of hyporheic exchange, in-stream transport, and reactivity parameters.

In the present study, the general residence time distribution model proposed by Marion et al. (2008) is extended to represent the transport and transformation of Raz-Rru tracers, and a new software tool, STIR-RST, is presented for inverse modelling of stream transport and reaction processes using smart tracer data. We present an application of STIR-RST to an experimental dataset from a field tracer study where transient storage is described by two storage domains with exponential RTD. In this application, we consider two alternative arrangements of the storage zones: competing (in parallel) and nested (in series). We discuss the calibrated model parameters in light of the properties of the study reaches and compare the uncertainty in the model parameters with a single-storage zone model. This application exemplifies how STIR-RST can be used in stream tracer studies to evaluate alternative transient storage assumptions and characterise physical stream processes.

\section{Model}

\subsection{Time domain formulation}

The transport of a solute along a river channel is represented as a stochastic process involving the motion of a large number of solute "particles". To derive the fundamental model equations, we initially assume that the transport within the main flow channel (MFC) is affected by trapping in a single storage zone (SZ), and later extend the theory to the case of multiple storage zones. We assume that any significant streamwise transport occurs only in the MFC and represent the storage zone as an immobile domain. 
Resazurin (Raz) is transformed into Resorufin (Rru) according to a first-order reaction with constant kinetic rate $\lambda_{W 12}$ in the main channel, and $\lambda_{S 12}$ in the storage zones. For sake of generality, we also consider the potential decay of the solute according to first-order kinetics with decay rates $\lambda_{W 1}$ and $\lambda_{S 1}$ for Resazurin, and $\lambda_{W 2}$ and $\lambda_{S 2}$ for Resorufin, where the subscripts $W$ and $S$ are associated with the MFC and the SZ, respectively, and the numbers refer to the tracer type.

Let $\psi_{C}(x, t) d x$ be the probability that a particle of passive solute has traveled a distance between $x$ and $x+d x$ in a time $t$ knowing that the particle has never been trapped in the SZ. The probability density that a particle of Resazurin never trapped in the SZ has travelled a distance $x$ without undergoing chemical transformations is:

$$
\psi_{C}(x, t) e^{-\left(\lambda_{W 1}+\lambda_{W 12}\right) t}
$$

where $e^{-\left(\lambda_{W 1}+\lambda_{W 12}\right) t}$ is the survival probability. The function $\psi_{C}$ is referred to as the probability density function (PDF), or distribution, of jump length or traveled distance in the MFC.

Every time a particle is trapped in the SZ, the residence time in the SZ is assumed to be distributed according to the PDF $\varphi(t)$. Assuming that the residence times associated with different trapping events are conditionally independent, the overall residence time distribution in the SZ for a particle trapped $n$ times along the MFC is:

$$
\varphi^{* n}(t)=\underbrace{\varphi(t) * \varphi(t) * \ldots * \varphi(t)}_{n \text { times }}
$$

where the symbol $*$ denotes convolution:

$$
\varphi(t) * \varphi(t)=\int_{0}^{t} \varphi(\tau) \varphi(t-\tau) d \tau
$$

The probability density that a Resazurin particle trapped in a storage zone is released from the storage zone after a time $t$ without undergoing chemical transformations is therefore:

$$
\varphi^{* n}(t) e^{-\left(\lambda_{S 1}+\lambda_{S 12}\right) t}
$$

Let $p(n \mid t)$ be the conditional probability that a particle is trapped $n$ times in the SZ knowing that the particle has spent a time $t$ in the MFC. The probability of a Resazurin particle travelling a distance $x$ within a time $t$ without chemically reacting is:

$$
\psi_{R a z}(x, t)=\sum_{n=0}^{\infty} \int_{0}^{t} \psi_{C}(x, \tau) e^{-\left(\lambda_{W 1}+\lambda_{W 12}\right) \tau} p(n \mid \tau) \varphi^{* n}(t-\tau) e^{-\left(\lambda_{S 1}+\lambda_{S 12}\right)(t-\tau)} d \tau
$$


If the probability that a particle is trapped along the MFC is spatially uniform and constant in time, and if the trapping events are conditionally independent, the probability $p(n \mid t)$ is described by the Poisson distribution (Marion et al., 2008):

$$
p(n \mid t)=\frac{(\alpha t)^{n} e^{\alpha t}}{n !}
$$

where $\alpha$ is the trapping probability per unit time, equivalent to a transfer rate.

We assume that the hydrodynamic processes affecting the transport of Resorufin are the same as for Resazurin, but the chemical transformations are different. Following similar derivations as those presented above, the probability that a particle of Resorufin travels a distance $x$ in a time $t$ without chemical transformation is:

$$
\psi_{R r u}^{\dagger}(x, t)=\sum_{n=0}^{\infty} \int_{0}^{t} \psi_{C}(x, \tau) e^{-\lambda_{W 2} \tau} p(n \mid \tau) \varphi^{* n}(t-\tau) e^{-\lambda_{S 2}(t-\tau)} d \tau
$$

The probability density per unit time that a particle of Resorufin is generated in the MFC at position $x$ at time $t$ is:

$$
g_{W}(x, t)=\lambda_{W 12} \psi_{R a z}(x, t)
$$

which is then transported according to equation (7). The probability density that a particle of Resazurin is transformed into Resorufin after spending a time $t$ in the SZ is:

$$
\lambda_{S 12} e^{-\lambda_{S 12} t}
$$

Therefore, if different decay rates are considered for Resazurin and Resorufin, the probability that a particle of Resazurin exiting the SZ as Resorufin at position $x$ after spending a time $t$ in a storage zone is:

$$
\varphi(t) \int_{0}^{t} \lambda_{S 12} e^{-\lambda_{S 12} \tau} e^{-\lambda_{S 1} \tau} e^{-\lambda_{S 2}(t-\tau)} d \tau=\varphi(t) \frac{\lambda_{S 12}\left(e^{-\lambda_{S 2} t}-e^{-\left(\lambda_{S 1}+\lambda_{S 12}\right) t}\right)}{\lambda_{S 1}+\lambda_{S 12}-\lambda_{S 2}}
$$

In the equation above the probability that a particle of Resazurin exits as Resorufin after spending a time $t$ in the storage zone is calculated as a joint probability that (i) the particle stays in a storage zone (SZ) for a time $t$ and (ii) the particle exits the SZ as Resorufin knowing that the particle has spent a time $t$ in the SZ. The latter is calculated as the integral from 0 to $t$ of the joint probability that: (i) a particle of Resazurin is not transformed into an unknown product (and therefore survives as Resazurin) between 0 and $\tau$; (ii) a particle is transformed to Resorufin between $\tau$ and $\tau+d \tau$; (iii) a particle of Resorufin is not transformed into an unknown product between $\tau$ and $t$.

The generation of Resorufin per unit time at position $x$ in the SZ time is therefore: 


$$
g_{S}(x, t)=\alpha \int_{0}^{t} \varphi(\tau) \frac{\lambda_{S 12}\left(e^{-\left(\lambda_{S 1}+\lambda_{S 12}\right) \tau}-e^{-\lambda_{S 2} \tau}\right)}{\lambda_{S 1}+\lambda_{S 12}-\lambda_{S 2}} \psi_{R a z}(x, t-\tau) d \tau
$$

Particles of Resorufin generated in the MFC and in the SZ are transported according to (7), hence:

$$
\psi_{R r u}(x, t)=\int_{0}^{t} \int_{-\infty}^{\infty}\left(g_{W}(\xi, \tau)+g_{S}(\xi, \tau)\right) \psi_{R r u}^{\dagger}(x-\xi, t-\tau) d \xi d \tau
$$

For a mass injection rate of Resazurin $\dot{M}_{R a z}(t)$ at point $x=0$, the concentrations of Resazurin, $C_{R a z}$, and Resorufin, $C_{R r u}$, are respectively given by:

$$
\begin{aligned}
& C_{R a z}(x, t)=\frac{1}{A} \int_{0}^{t} \dot{M}_{R a z}(\tau) \psi_{R a z}(x, t-\tau) d \tau \\
& C_{R r u}(x, t)=\frac{1}{A} \int_{0}^{t} \dot{M}_{R a z}(\tau) \psi_{R r u}(x, t-\tau) d \tau
\end{aligned}
$$

Alternatively, for a concentration of Resazurin specified at the upstream boundary, $C_{R a z, b}(t)$, one needs to look at the probability that a solute particle originally at the boundary travels a distance $x$ in a time between $t$ and $t+d t$. This is referred to as transit time probability or residence time probability. Indicating with $r_{C}(x, t)$ the transit time PDF for a segment of the MFC of length $x$ in the absence of storage and reactions, and with $r_{R a z}(x, t)$ the transit time PDF for Resazurin, the expression for $r_{R a z}(x, t)$ is still given by (5), but with $\psi_{C}$ now replaced by $r_{C}$. Similarly, if the transit time PDF for Resorufin is denoted by $r_{R r u}$, and $r_{R r u}^{\dagger}$ is the transit time PDF counterpart of $\psi_{R r u}^{\dagger}$ obtained by replacing $\psi_{C}$ with $r_{C}$ in (7), then $r_{R r u}$ is given by (12) with $\psi_{R r u}^{\dagger}$ replaced by $r_{R r u}^{\dagger}$. The concentrations $C_{R a z}(x, t)$ and $C_{R r u}(x, t)$ can now be expressed as follows:

$$
\begin{aligned}
& C_{R a z}(x, t)=\int_{0}^{t} C_{R a z, b}(\tau) r_{R a z}(x, t-\tau) d \tau \\
& C_{R r u}(x, t)=\int_{0}^{t} C_{R a z, b}(\tau) r_{R r u}(x, t-\tau) d \tau
\end{aligned}
$$

As a special case, it can be assumed that the transport of a passive solute in the MFC is described by the advection-dispersion equation (ADE):

$$
\frac{\partial C}{\partial t}+U \frac{\partial C}{\partial x}-D \frac{\partial^{2} C}{\partial x^{2}}=0
$$

where $C$ is concentration, $U$ is flow velocity, $D$ is the longitudinal dispersion coefficient. The ADE implies that the distribution of jump length is 


$$
\psi_{C}(x, t)=\frac{1}{2 \sqrt{\pi D t}} e^{-\frac{(x-U t)^{2}}{4 D t}}
$$

and the transit time distribution is

$$
r_{C}(x, t)=\frac{x}{2 \sqrt{\pi D t^{3}}} e^{-\frac{(x-U t)^{2}}{4 D t}}
$$

If the storage zone has finite cross-sectional area, $A_{S}$, and the interfacial transfer rate, $\alpha$, is constant, the residence time follows an exponential distribution,

$$
\varphi(t)=\frac{1}{T} e^{-t / T}
$$

where $T=\alpha A / A_{S}$ is the average residence time.

\subsection{Laplace domain formulation}

The evaluation of the convolution integrals that appear in the time domain formulation can be simplified by using Laplace transform (LT). The LT of a function $\varphi(t)$ is defined as:

$$
\tilde{\varphi}(s)=\int_{0}^{\infty} \varphi(t) e^{-s t} d t
$$

where $s$ is the Laplace variable. Using the convolution theorem, the LT of (5) can be written as:

$\tilde{\psi}_{R a z}(x, s)=\sum_{n=0}^{\infty}\left(\int_{0}^{\infty} \psi_{C}(x, t) e^{-\left(\lambda_{W 1}+\lambda_{W 12}\right) t} p(n \mid t) e^{-s t} d t\right) \tilde{\varphi}^{n}\left(s+\lambda_{s 1}+\lambda_{s 12}\right)$

If the trapping probability follows a Poisson distribution, combining (6) with (22) gives:

$$
\tilde{\psi}_{R a z}(x, s)=\sum_{n=0}^{\infty} \int_{0}^{\infty} \psi_{C}(x, t) \frac{\left[\alpha \tilde{\varphi}\left(s+\lambda_{S 1}+\lambda_{S 12}\right) t\right]^{n}}{n !} e^{-\left(s+\lambda_{W 1}+\lambda_{W 12}-\alpha\right) t} d t
$$

By using the identity

$$
\frac{\left[\alpha \tilde{\varphi}\left(s+\lambda_{s 1}+\lambda_{s 12}\right) t\right]^{n}}{n !}=e^{\alpha \widetilde{\varphi}\left(s+\lambda_{S 1}+\lambda_{s 12}\right) t}
$$

and the shifting theorem of Laplace transforms, equation (24) gives:

$$
\begin{aligned}
\tilde{\psi}_{R a z}(x, s) & =\tilde{\psi}_{C}\left(x, s+\lambda_{W 1}+\lambda_{W 12}+\alpha\left(1-\tilde{\varphi}\left(s+\lambda_{S 1}+\lambda_{S 12}\right)\right)\right) \\
& =\tilde{\psi}_{C}\left(x, v_{R a z}(s)\right)
\end{aligned}
$$

where

$$
v_{R a z}(s)=s+\lambda_{W 1}+\lambda_{W 12}+\alpha\left(1-\tilde{\varphi}\left(s+\lambda_{S 1}+\lambda_{S 12}\right)\right)
$$

is a frequency variable. Similarly, the LT of $\psi_{R r u}^{\dagger}(x, t)$ can be written as: 


$$
\begin{aligned}
\tilde{\psi}_{R r u}^{\dagger}(x, s) & =\tilde{\psi}_{C}\left(x, s+\lambda_{W 2}+\alpha\left(1-\tilde{\varphi}\left(s+\lambda_{s 2}\right)\right)\right) \\
& =\tilde{\psi}_{C}\left(x, v_{R r u}(s)\right)
\end{aligned}
$$

where

$$
v_{R r u}(s)=s+\lambda_{W 2}+\alpha\left(1-\tilde{\varphi}\left(s+\lambda_{S 2}\right)\right)
$$

The LTs of the generation functions $g_{W}$ and $g_{S}$ are:

$$
\tilde{g}_{W}(s)=\lambda_{W 12} \tilde{\psi}_{R a z}(x, s)
$$

and

$$
\tilde{g}_{S}(x, s)=\alpha \frac{\tilde{\varphi}\left(s+\lambda_{S 2}\right)-\tilde{\varphi}\left(s+\lambda_{S 1}+\lambda_{S 12}\right)}{\lambda_{S 1}+\lambda_{s 12}-\lambda_{S 2}} \tilde{\psi}_{R a z}(x, s)
$$

respectively. Hence:

$$
\begin{aligned}
& \tilde{\psi}_{R r u}(x, s) \\
& =\left[\lambda_{W 12}+\alpha \frac{\tilde{\varphi}\left(s+\lambda_{S 2}\right)-\tilde{\varphi}\left(s+\lambda_{S 1}+\lambda_{s 12}\right)}{\lambda_{S 1}+\lambda_{S 12}-\lambda_{S 2}}\right] \int_{-\infty}^{\infty} \tilde{\psi}_{R a z}(\xi, s) \tilde{\psi}_{R r u}^{\dagger}(x-\xi, s) d \xi \\
& =\left[\lambda_{W 12}+\alpha \frac{\tilde{\varphi}\left(s+\lambda_{S 2}\right)-\tilde{\varphi}\left(s+\lambda_{S 1}+\lambda_{S 12}\right)}{\lambda_{S 1}+\lambda_{S 12}-\lambda_{S 2}}\right] \int_{-\infty}^{\infty} \tilde{\psi}_{C}\left(\xi, v_{R a z}(s)\right) \tilde{\psi}_{C}\left(x-\xi, v_{R r u}(s)\right) d \xi
\end{aligned}
$$

As a special case, if the distribution (18) resulting from the classic ADE is assumed to represent the transport in the MFC, and the exponential distribution (20) is assumed for the SZ, the LT of (5) and (12) are, respectively:

$$
\begin{gathered}
\tilde{\psi}_{R a z}(x, s)=\frac{e^{\frac{x\left(U-\sqrt{4 D v_{R a z}(s)+U^{2}}\right)}{2 D}}}{\sqrt{4 D v_{R a z}(s)+U^{2}}} \\
\tilde{\psi}_{R r u}(x, s)=\left[\lambda_{W 12}+\alpha \frac{\lambda_{S 12} T}{\left(1+s T+\left(\lambda_{S 1}+\lambda_{S 12}\right) T\right)\left(1+s T+\lambda_{S 2} T\right)}\right] \\
\frac{\sqrt{4 D v_{R a z}(s)+U^{2}} e^{\frac{x\left(U-\sqrt{4 D v_{R r u}(s)+U^{2}}\right)}{2 D}}-\sqrt{4 D v_{R r u}(s)+U^{2}} e^{\frac{x\left(U-\sqrt{4 D v_{R a z}(s)+U^{2}}\right)}{2 k}}}{\left[v_{R a z}(s)-v_{R r u}(s)\right] \sqrt{4 D v_{R a z}(s)+U^{2}} \sqrt{4 D v_{R r u}(s)+U^{2}}}
\end{gathered}
$$

where:

$$
\begin{gathered}
v_{R a z}(s)=s+\lambda_{W 1}+\lambda_{W 12}+\alpha\left[1-\frac{1}{1+\left(s+\lambda_{S 1}+\lambda_{S 12}\right) T}\right] \\
v_{R r u}(s)=s+\lambda_{W 2}+\alpha\left[1-\frac{1}{1+\left(s+\lambda_{S 2}\right) T}\right]
\end{gathered}
$$




\subsection{Exchange with multiple storage zones}

The model equations derived above can be easily extended to the case of multiple zones in parallel. The expression for $\tilde{\psi}_{R a z}$ remains the same (equation (25)) but now the frequency variable $v_{R a z}(s)$ is:

$$
v_{R a z}(s)=s+\lambda_{W 1}+\lambda_{W 12}+\sum_{i=0}^{N_{S}} \alpha_{i}\left(1-\tilde{\varphi}_{i}\left(s+\lambda_{s 1, i}+\lambda_{s 12, i}\right)\right)
$$

where $N_{S}$ is the number of storage zones. Taking into account the generation of Resorufin in the SZs, the expression for $\tilde{\psi}_{R r u}$ becomes:

$$
\begin{aligned}
\tilde{\psi}_{R r u}(x, s)= & {\left[\lambda_{W 12}+\sum_{i=0}^{N_{S}} \alpha_{i} \frac{\tilde{\varphi}_{i}\left(s+\lambda_{S 2, i}\right)-\tilde{\varphi}_{i}\left(s+\lambda_{S 1, i}+\lambda_{S 12, i}\right)}{\lambda_{S 1, i}+\lambda_{S 12, i}-\lambda_{S 2, i}}\right] . } \\
& \int_{-\infty}^{\infty} \tilde{\psi}_{C}\left(\xi, v_{R a z}(s)\right) \tilde{\psi}_{C}\left(x-\xi, v_{R r u}(s)\right) d \xi
\end{aligned}
$$

where:

$$
v_{R r u}(s)=s+\lambda_{W 2}+\sum_{i=0}^{N_{S}} \alpha_{i}\left(1-\tilde{\varphi}_{i}\left(s+\lambda_{S 2, i}\right)\right)
$$

Alternatively, we can consider the case of two storage zones in series, with the transfer rate $\alpha_{2}$ representing the probability per unit time that a solute particle in the first SZ (denoted with the subscript 1 in the derivations that follow) can be trapped in the second SZ (denoted with the subscript 2). Similar considerations to those presented above for the exchange between the MFC and the SZ now apply to the exchange between the first and the second SZ. The PDF $\tilde{\psi}_{R a z}(x, s)$ is still given by (25), but now the frequency variable $v_{R a z}(s)$ is:

$$
v_{R a z}(s)=s+\lambda_{W 1}+\lambda_{W 12}+\alpha_{1}\left(1-\tilde{\varphi}_{1}\left(v_{R a z, 1}(s)\right)\right.
$$

where:

$$
v_{R a z, 1}(s)=s+\lambda_{s 1,1}+\lambda_{s 12,1}+\alpha_{2}\left(1-\tilde{\varphi}_{2}\left(s+\lambda_{s 1,2}+\lambda_{s 12,2}\right)\right)
$$

By modelling the exchange between the first and the second SZ in the same way as the exchange between the MFC and the SZ, we obtain:

$\tilde{g}_{S}(s)$
$=\left[\lambda_{S 12,1}+\alpha_{2} \frac{\tilde{\varphi}_{2}\left(s+\lambda_{S 2,2}\right)-\tilde{\varphi}_{2}\left(s+\lambda_{S 1,2}+\lambda_{S 12,2}\right)}{\lambda_{S 1,2}+\lambda_{S 12,2}-\lambda_{S 2,2}}\right] \tilde{\varphi}_{1}\left(v_{R r a, 1}(s)\right) \tilde{\varphi}_{1}\left(v_{R r u, 1}(s)\right)$

and 


$$
\tilde{\psi}_{R r u}(x, s)=\left[\lambda_{W 12}+\tilde{g}_{S}(s)\right] \int_{-\infty}^{\infty} \tilde{\psi}_{C}\left(\xi, v_{R a z}(s)\right) \tilde{\psi}_{C}\left(x-\xi, v_{R r u}(s)\right) d \xi
$$

where:

$$
\begin{gathered}
v_{R r u}(s)=s+\lambda_{W 2}+\alpha_{1}\left(1-\tilde{\varphi}_{1}\left(v_{R r u, 1}(s)\right)\right. \\
v_{R r u, 1}(s)=s+\lambda_{s 2,1}+\alpha_{2}\left(1-\tilde{\varphi}_{2}\left(s+\lambda_{s 2,2}\right)\right)
\end{gathered}
$$

\section{The STIR-RST software}

The stochastic formulation presented above provides a general modelling framework in which different storage processes can be represented by specific residence time distributions. This formulation was implemented in a Matlab toolbox which is released under the name STIRRST, an acronym for "Solute Transport In Rivers - Reactive Smart Tracer". The objectoriented structure of the STIR-RST toolbox allows the implementation of transport models incorporating different RTDs in the main channel and the storage zones as subclasses of the base model class StirRST. This superclass provides all the methods for handling model input and output data and parameter calibration, with the option of simulating the simultaneous transport of two tracers: a conservative tracer, or a tracer that decays into unknown products, and a "smart" tracer couple in which one tracer transforms into the other. The implementation of a transient storage model within the general framework of STIR-RST requires specification of the RTD functions for the MFC and the storage zones, and the definition of a mapping between the parameters defined in a template XML file and the arrays of input arguments used by the RTD functions. The definition of this mapping is facilitated by computer code that automatically translates the XML template files into data structures.

STIR-RST uses a semi-analytical approach in which the solute breakthrough curves in the time domain are obtained by numerical inversion of the Laplace transforms of the RTD functions, therefore the RTDs must be provided as functions of the Laplace variable $s$. In the present version of the toolbox, this is exemplified by the model class StirRST2ExpModel, which assumes exchange with two distinct storage zones, each characterized by an exponential residence time distribution, whereas the transport in the MFC in the absence of storage is represented by the classic advection-dispersion equation. Alternative descriptions of transient storage may be added to the toolbox in the future.

The simulation parameters can be read from an XML file or entered from the command line or a Matlab script. A template input file for the two-exponential RTD model is provided 
with the software and includes a description for each of the parameters. Running the model and visualizing the results involves the following sequence of instructions:

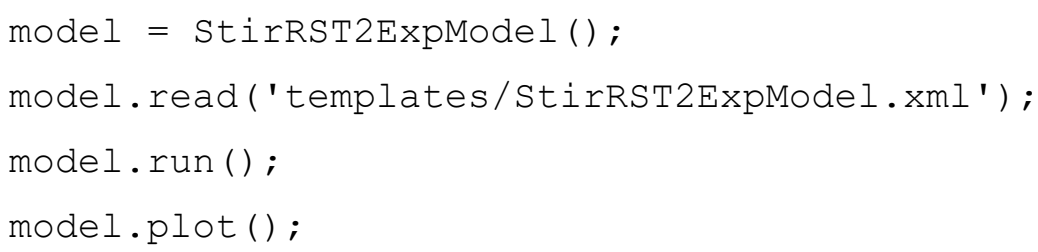

The software also allows model calibration using a range of optimization methods for local and global optimization, including the Nelder-Mead method, simulated annealing, particle swarm, pattern search, and differential evolution. After loading the concentration data in the Matlab workspace, the model can be calibrated with the instruction:

model.calibrate ()

An example script for model evaluation and calibration is provided with the software. It is possible to define which model parameters should be calibrated and their range of variation by modifying an additional file containing the calibration settings, or from the command line as in the example script provided. After calibration, the parameter values can then be listed on screen,

model.printParameters ()

or saved to an XML file:

model.write ('output.xml')

The user can choose between calibration in linear, logarithmic and mixed scale. In the case of linear scale calibration, the fitting error $\epsilon$ is given by the normalized root mean square deviation:

$$
\epsilon_{\text {lin }}=\sqrt{\frac{1}{N_{\mathrm{obs}}} \sum_{j=1}^{N_{\mathrm{obs}}} \frac{\left(C_{j}-C_{\mathrm{obs}, j}\right)^{2}}{\left(C_{\mathrm{obs}, \max }-C_{\mathrm{obs}, \min }\right)^{2}}}
$$

where $C_{j}$ are the simulated concentration values, $C_{\mathrm{obs}, j}$ are the observations, $C_{\mathrm{obs}, \max }$ is the maximum observed concentration value, and $C_{\mathrm{obs} \text {,min }}$ is the minimum observed value. For logarithmic scale,

$$
\epsilon_{\text {log }}=\sqrt{\frac{1}{N_{\mathrm{obs}}} \sum_{j=1}^{N_{\mathrm{obs}}} \frac{\left(\log C_{j}-\log C_{\mathrm{obs}, j}\right)^{2}}{\left(\log C_{\mathrm{obs}, \max }-\log C_{\mathrm{obs}, \min }\right)^{2}}}
$$

In the application of (46), the STIR-RST software allows to exclude the lowest percentile of the observed concentrations. The default value is $5 \%$, but can be modified from the software 
calibration options. For mixed scale, the error is calculated as proposed by Bottacin-Busolin et al. (2011):

$$
=\sqrt{\frac{1}{N_{\mathrm{obs}}}\left(\sum_{j \in I_{U}} \frac{\left(C_{j}-C_{\mathrm{obs}, j}\right)^{2}}{\left(C_{\mathrm{obs}, \max }-C_{\mathrm{obs}, \min }\right)^{2}}+\sum_{j \in I_{L}} \frac{\left(\log C_{j}-\log C_{\mathrm{obs}, j}\right)^{2}}{\left(\log C_{\mathrm{obs}, \max }-\log C_{\mathrm{obs}, \min }\right)^{2}}\right)}
$$

where $I_{U}$ and $I_{L}$ are the sets of observed values respectively higher and lower than a threshold concentration. The threshold value is defined in the STIR-RST software as a percentage of the peak concentration. The default value of the threshold is $20 \%$, but can be modified from the calibration options. As for the logarithmic scale calibration, the expression (47) is applied by excluding a given percentage of the lowest concentrations.

For smart tracer studies, the Resazurin and Resorufin breakthrough curves are fit simultaneously by extending the summation in the above definitions of the RMSE to the observed concentrations of Resazurin and Resorufin. Optionally, the software allows to simultaneously fit breakthrough curves from a conservative tracer and a smart tracer.

\section{Application}

In this section, an application of STIR-RST is presented where the model parameters are calibrated from field tracer data, considering two different arrangements of the storage zones, i.e., in series and in parallel.

\subsection{Experimental Test}

The study site is located in the countryside around the urban settlement of Monselice (Padua, Italy). The field experiments were carried out in the Desturo canal, a small drainage canal, about $6 \mathrm{~km}$ long, used for irrigation purposes. The canal has been affected by pollution due to distributed inputs of agricultural fertilizers, the discharge of waters from a wastewater treatment plant, and the discharge of untreated storm water from the surrounding urban area. The study reach was located downstream of the outfall of the water treatment plant, and had a length of $220 \mathrm{~m}$. The injection point (I0) was located just downstream of the outfall to ensure rapid mixing of the injected tracer. Two measurement stations (St1 and St2) were located at distances $L_{01}=130 \mathrm{~m}$ and $L_{02}=220 \mathrm{~m}$ from the injection point I0, respectively. We refer to the reach from I0 to St1 as R01, from I0 to St2 as R02, and from St1 to St2 as R12. From direct measurements, we estimated the average depth and width of reach R01 to be $h=0.44 \mathrm{~m}$ and $b=2.7 \mathrm{~m}$, respectively, corresponding to an average cross-sectional area $A_{01}=1.18 \mathrm{~m}^{2}$. For 
reach $\mathrm{R} 02$, the average flow depth and width were estimated as $h=0.36 \mathrm{~m}$ and $b=2.6 \mathrm{~m}$, respectively, and therefore the average flow cross-sectional area was $A_{02}=0.93 \mathrm{~m}^{2}$. During the tests, flow measurements were taken using a current meter, from which the discharge was subsequently estimated as $Q=0.054 \mathrm{~m}^{3} \mathrm{~s}^{-1}$. An estimate of the distance from the injection point required to achieve complete transverse mixing was obtained from the average bed slope of the channel in the study area, which was estimated from topographical survey as $S=0.17 \%$. This corresponds to a shear velocity $u_{*}=\sqrt{g h S}=0.027 \mathrm{~m} \mathrm{~s}^{-1}$ and a reference transverse mixing coefficient for a straight rectangular channel (Fischer et al., 1979) $\varepsilon_{t}=0.15 u_{*} h=$ $1.8 \times 10^{-3} \mathrm{~m}^{2} \mathrm{~s}^{-1}$ based on the flow depth for the first study reach. The resulting mixing length for a central injection is $L_{m}=\frac{U b^{2}}{4 \varepsilon_{t}}=47 \mathrm{~m}$, where $U=Q / A$ is the average velocity. We can thus conclude that the first measuring station was located sufficiently far away from the injection point to ensure complete mixing over the channel cross-section.

The whole study reach was characterized by sandy-silty material, with submerged vegetation mostly along $\mathrm{R} 12$. The study reach R01 had an almost constant width, except for a localized enlargement downstream of a $90^{\circ}$ bend. Vegetation on the banks was quite thin along R01, with few short spots of concrete or rock. Reach 12 was characterized by an almost straight channel, with much more vegetation along the banks and the bed. Map of the study site and the location of the measurement stations are given in Figure 1, and some photos taken along the study reach are shown in Figure 2.
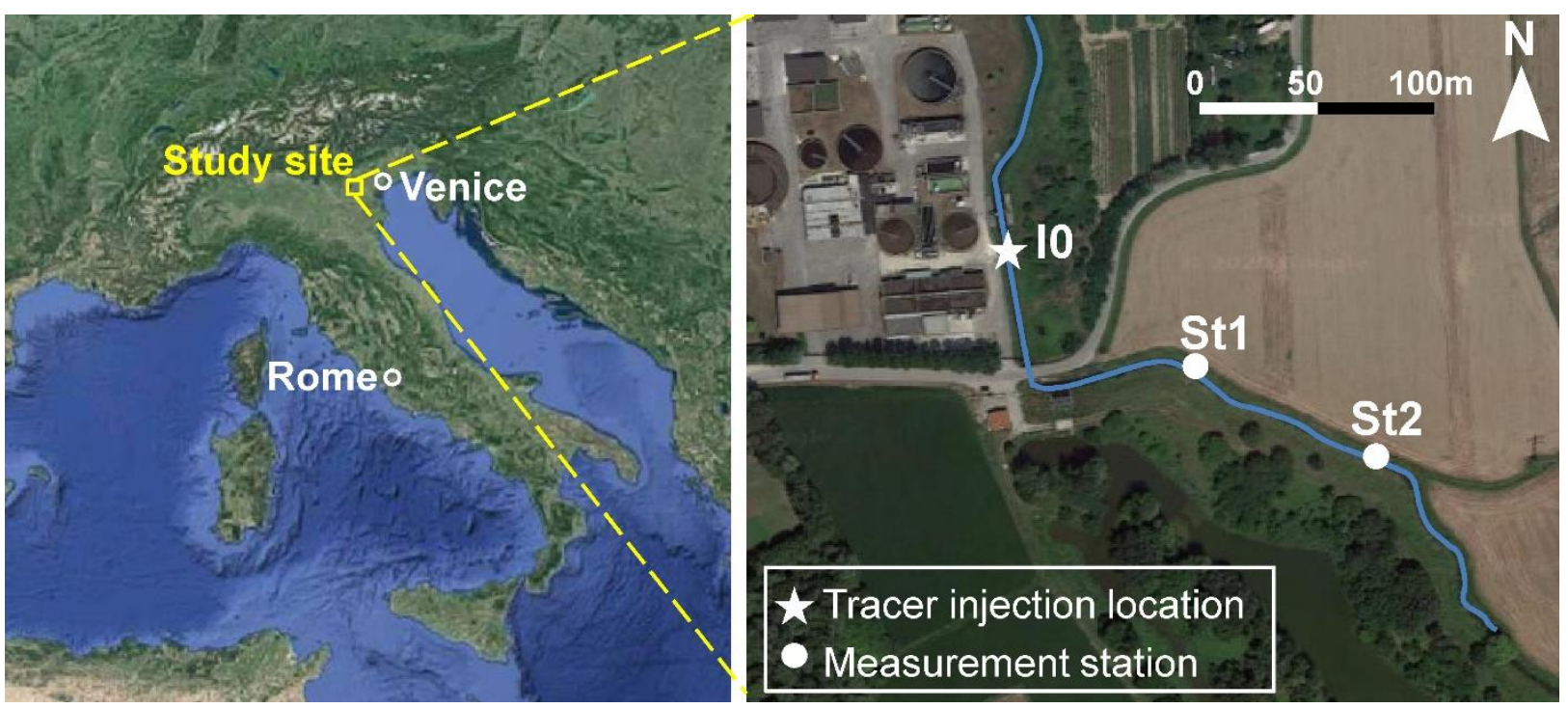

Figure 1 Study area in Monselice (Padua, Italy) and location of injection and gauge stations along the Desturo Canal downstream of a treatment plant. 

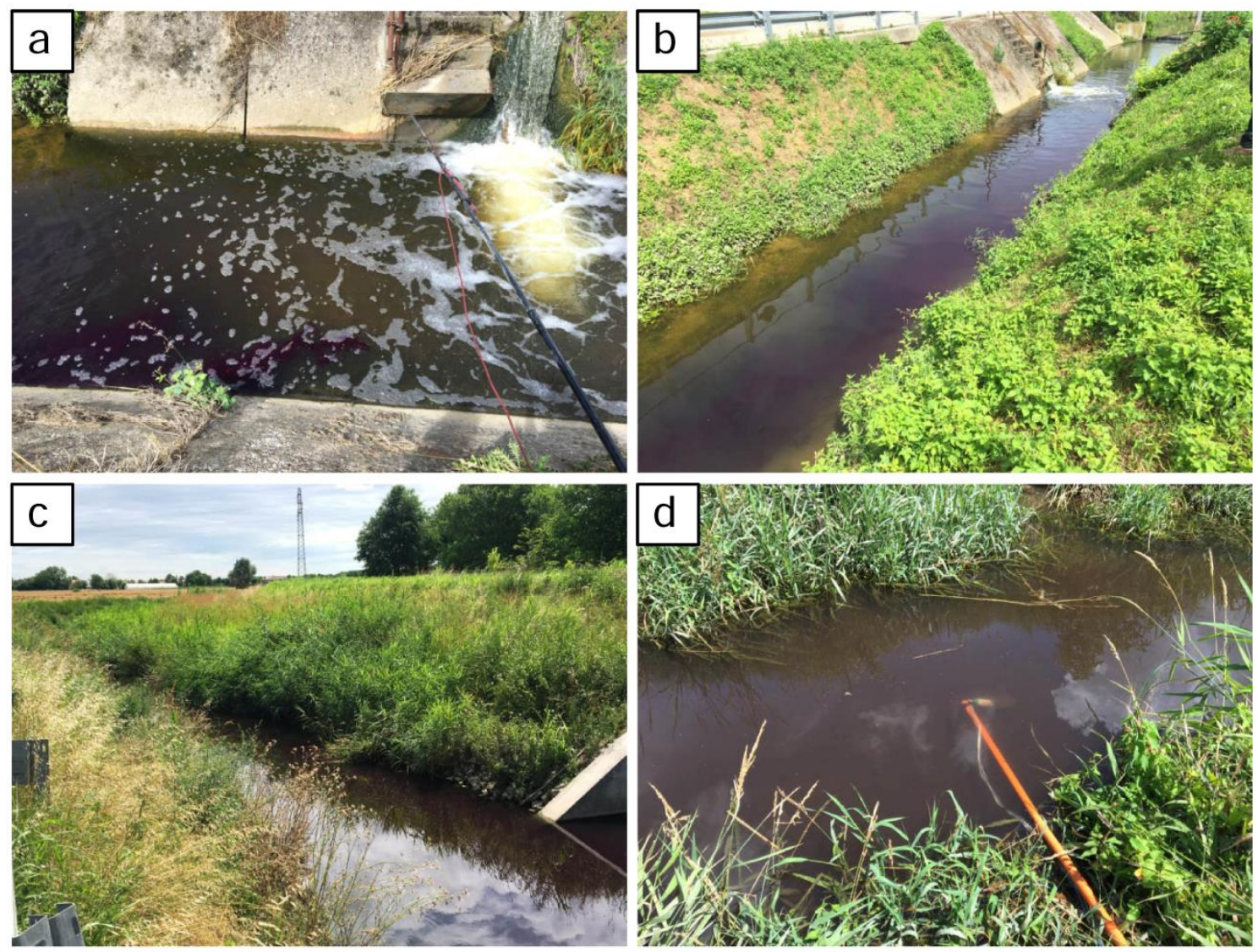

Figure 2 Canal characteristics along the study reach: a) station I0 and release of tracer downstream of the outlet of the treatment plant; b) initial straight segment of reach R01, characterized by clean banks and bed; c) reach R01 downstream of the bend, and initial part of reach R12 (more vegetated); d) measurement station S2; submerged vegetation can be seen along the river side.

The tracer test was carried out in June 2018. During the test, a solution of Raz and water with Raz concentration of $10 \mathrm{~g} \mathrm{~L}^{-1}$ was injected at station I0 (Figure 2a) with a constant rate for 22 minutes using a peristaltic pump, resulting in a total injection of $14.30 \mathrm{~g}(0.057 \mathrm{~mol})$ of Raz. At the two stations $\mathrm{St} 1$ and $\mathrm{St} 2$, fluorescence intensity signals were recorded at a sampling period of 20 s using two GGUN-FL30 on-line fluorometers (Albilia Sarl, Switzerland), with typical detection limit of $2 \times 10^{-3} \mathrm{Mg} \mathrm{L}^{-1}$, for about 4 hours. These are flow-through fluorometers that allow simultaneous detection of three tracers, as well as measurements of turbidity and water temperature. Recorded intensity signals were converted to dye concentration values after calibration with the software provided by the manufacturer. The baseline signal before injection was subtracted from the time series. Two breakthrough curves were obtained at each station, representing Raz and Rru concentrations over time. The on-line fluorometer allowed a highresolution sampling of tracer concentrations over several hours. Figure 3 shows the BTCs both in linear and semi-log scale. It can be seen that there is a clear decrease in Raz peak concentration between sections St 1 and St2, and a small increase of the concentration peak for Rru, while the tail of the Rru BTC is longer at section St2. This suggests that retention processes 
are more effective in reach R12 (downstream of St1), resulting in higher Rru production and longer BTC tails at section St2.
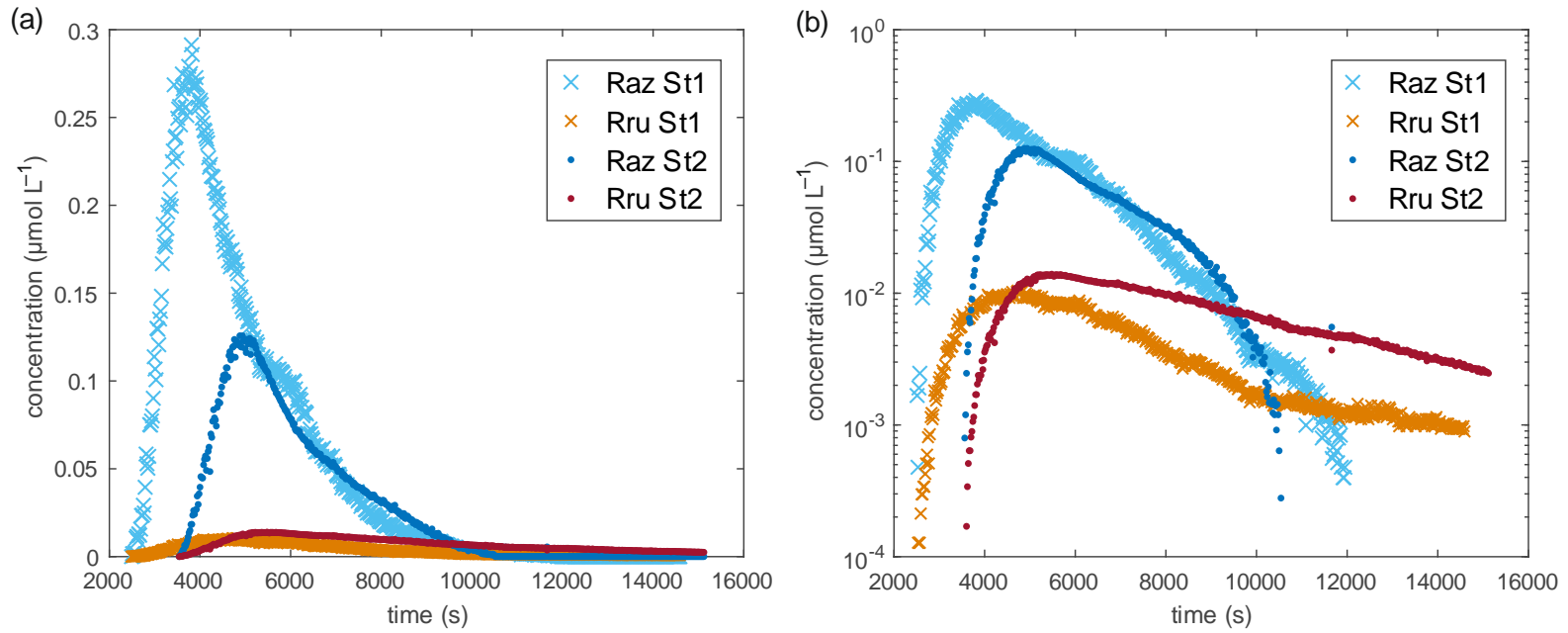

Figure 3 Concentration curves measured at the two stations, St1 and St2, for Raz and Rru in (a) linear scale and (b) semi-log scale.

\subsection{Model Calibration}

In this example application of STIR-RST, we assumed exponential distribution of the residence time in two distinct storage domains with two alternative arrangements of the storage zones, i.e., in parallel and in series, and also compared the results with the case of a single storage zone with exponential RTD. We refer to these three special cases of the general model formulation presented in section 2 as 2SZ-P, 2SZ-S, and 1SZ, respectively. Table 1 presents the complete list of calibration parameters for the two-storage zone model. As mentioned above, flow cross-sectional area $A$ and discharge $Q$ were estimated from field measurements.

Table 1. Calibration parameters of the two-storage zone model with zones in parallel (2SZ-P) and in series (2SZ-S). The single-storage zone model (1SZ) considered in this work has the same storage and reactivity parameters, but only for one storage zone.

\begin{tabular}{llc}
\hline Symbol & Description & Units \\
\hline$D$ & Dispersion coefficient & $\mathrm{m}^{2} \mathrm{~s}^{-1}$ \\
$\lambda_{\mathrm{W} 1}, \lambda_{\mathrm{W} 2}$ & Decay rates for Raz (1), Rru (2) in the main channel & $\mathrm{s}^{-1}$ \\
$\lambda_{\mathrm{S} 1,1}, \lambda_{\mathrm{S} 2,1}$ & Decay rates for Raz (1), Rru (2) in the storage zone \#1 & $\mathrm{s}^{-1}$ \\
$\lambda_{\mathrm{S} 1,2}, \lambda_{\mathrm{S} 2,2}$ & Decay rates for Raz (1), Rru (2) in the storage zone \#2 & $\mathrm{s}^{-1}$ \\
$\lambda_{\mathrm{W} 12} \lambda_{\mathrm{S} 12,1}, \lambda_{\mathrm{S} 12,2}$ & Transformation rates from Raz to Rru, in the main channel (W), SZ1 (1), & $\mathrm{s}^{-1}$ \\
$T_{1}$ & SZ2 (2) & $\mathrm{s}$ \\
$T_{2}$ & Mean residence time for the storage zone \#1 & $\mathrm{s}$ \\
$\alpha_{1}$ & Mean residence time for the storage zone \#2 & $\mathrm{s}^{-1}$ \\
$\alpha_{2}$ & Exchange rate between storage zone \#1 and MFC & $\mathrm{s}^{-1}$ \\
& $\begin{array}{l}\text { Exchange rate between storage zone \#2 and MFC (for in-parallel } \\
\text { arrangement), or storage zone \#2 and storage zone \#1 (for in-series }\end{array}$ \\
\hline
\end{tabular}


Model input data include concentrations of Raz and Rru in the time at sections St1 and St2, injection duration $T_{\text {Raz,inj }}=1320 \mathrm{~s}$, injected Raz mass $M_{\text {Raz,inj }}=0.057 \mathrm{~mol}$, discharge $Q=$ $0.054 \mathrm{~m}^{3} \mathrm{~s}^{-1}$, averaged cross-sectional areas $A_{01}=1.18 \mathrm{~m}^{2}$ for reach $\mathrm{R} 01$ and $A_{02}=$ $0.93 \mathrm{~m}^{2}$ for reach $\mathrm{R} 02$, reach lengths $L_{01}=130 \mathrm{~m}$ for reach R01, and $L_{02}=220 \mathrm{~m}$ for reach R02. The calibration parameters include: dispersion coefficient $D$; exchange rates $\alpha_{1}$ and $\alpha_{2}$; mean residence times $T_{1}$ and $T_{2}$; rates of Raz decay, Raz to Rru transformation, and Rru decay in the MFC, i.e., $\lambda_{\mathrm{W} 1}, \lambda_{\mathrm{W} 12}, \lambda_{\mathrm{W} 2}$, respectively; rates of Raz decay, Raz to Rru transformation, Rru decay in the first storage zone $\mathrm{SZ} 1$, i.e., $\lambda_{\mathrm{S} 1,1}, \lambda_{\mathrm{S} 12,1}, \lambda_{\mathrm{S} 2,1}$, respectively; rates of Raz decay, Raz to Rru transformation, Rru decay in the second storage zone SZ2, i.e., $\lambda_{\mathrm{S} 1,2}, \lambda_{\mathrm{S} 2,2}, \lambda_{\mathrm{s} 12,2}$, respectively.

For both reaches, the parameters were estimated by minimizing the mixed-scale error $\epsilon_{m}$ (47) in two stages: first, by using the Particle Swarm method for global optimization, and subsequently by using the Nelder-Mead method to obtain a refined estimate of the best-fit parameters. Confidence intervals at the $95 \%$ level were obtained using a Markov chain Monte Carlo (MCMC) method assuming uniform prior distribution of the parameters and a multiGaussian likelihood function in the form:

$$
\mathcal{L}\left(\boldsymbol{C}_{\mathrm{obs}} \mid \boldsymbol{\theta}\right)=\prod_{j=1}^{N_{\mathrm{obs}}} \frac{1}{\sqrt{2 \pi \sigma_{j}^{2}}} \exp \left\{-\frac{\left(C_{j}(\boldsymbol{\theta})-C_{\mathrm{obs}, j}\right)^{2}}{2 \sigma_{j}^{2}}\right\}
$$

where $C_{j}(\boldsymbol{\theta})$ are the simulated concentrations for the parameter vector $\boldsymbol{\theta}$, and where the variance $\sigma_{j}^{2}$ is estimated as $\sigma_{j}^{2}=\frac{1}{N_{\mathrm{obs}}} \sum_{j \in I_{U}}\left(C_{j}(\widehat{\boldsymbol{\theta}})-C_{\mathrm{obs}, j}\right)^{2}$ for $j \in I_{U}$, where $\widehat{\boldsymbol{\theta}}$ is the optimal parameter vector, and likewise for $j \in I_{L}$. Note that this choice of the variance $\sigma_{j}^{2}$ differs from other studies which have assumed $\sigma_{j}^{2}$ to be the measurement error based on measurement device performance (e.g. Rana et al., 2019; Lemke et al., 2013), and implies that the likelihood of the parameter values is evaluated with respect to the best-fit error. An adaptive Metropolis algorithm (Haario et al., 2006) was used to sample from the posterior distribution and generate chains of 500,000 samples with a burnin phase of 20,000 generations.

\subsection{Results and Discussion}

\subsubsection{Simulated BTCs and Recovered Mass}

The breakthrough curves (BTCs) simulated with the single-storage zone and the twostorage zone models are compared with the observed BTCs in Figure 4. Both the 1SZ and 2SZ 
models provide reasonably good fits of the BTCs, but the 1SZ model does not well represent the tail behaviour of the Rru curves, resulting in a significantly higher error $\epsilon_{m}$. In section St1 the observed BTC of Raz exhibits a slight hump in the decreasing part of the curve, after the peak, at approximately $6000 \mathrm{~s}$, which cannot be reproduced by the model. For the Raz BTC at section St2, the fit is poorer at around $8000 \mathrm{~s}$, where the simulated BTC decreases faster than the measured one. At section St1 the model fit is better than that at section St2, and this corresponds to a lower value of the mixed-scale error $\epsilon_{m}$ estimated according to (47). In this application, the quality of the fit is similar for in-series and in-parallel arrangements, with practically overlapping simulated curves.

The total mass recovered, $M$, at the two sections is calculated for each breakthrough curve as follows:

$$
M=Q \int_{t_{0}}^{t_{f}} C(t) d t
$$

where $t_{0}$ is the initial observation time, $t_{f}$ is the final observation time, and the integral represents the zero-th moment of the breakthrough curve. The results are summarized in Table 2. For section St1, the total recovered mass $M_{\text {tot }}$ deduced from the observed BTC is about $68 \%$ of the injected mass $M_{R a z, i n j}$. The simulations with the $1 \mathrm{SZ}$ and $2 \mathrm{SZ}$ models have a slightly lower mass recovery, with an error of approximately $0.6 \%$ and $2.3 \%$, respectively. For section $\mathrm{St} 2$, the total recovered mass is around $40 \%$, which is again slightly underestimated by the $1 \mathrm{SZ}$ model, with an error of $1.8 \%$, and the $2 \mathrm{SZ}$ model, with an error of $0.5 \%$.

Table 2. Mass recovered.

\begin{tabular}{|c|c|c|c|c|c|c|c|c|}
\hline \multirow{3}{*}{$\begin{array}{l}\text { Section } \\
\text { Dataset } \\
\text { Model }\end{array}$} & \multirow{3}{*}{$\frac{\text { St1 }}{\text { measured }}$} & \multicolumn{7}{|c|}{ St2 } \\
\hline & & \multicolumn{3}{|c|}{ calculated } & \multirow[t]{2}{*}{ measured } & \multicolumn{3}{|c|}{ calculated } \\
\hline & & $1 \mathrm{SZ}$ & 2SZ-S & 2SZ-P & & 1-SZ & 2SZ-S & 2SZ-P \\
\hline$M_{R a z}\left(\times 10^{-3} \mathrm{~mol}\right)$ & 36.42 & 35.1 & 36.1 & 36.1 & 18.3 & 17.7 & 18.0 & 18.0 \\
\hline$M_{R r u}\left(\times 10^{-3} \mathrm{~mol}\right)$ & 2.50 & 2.49 & 2.48 & 2.48 & 4.50 & 4.01 & 4.46 & 4.47 \\
\hline$M_{t o t}\left(\times 10^{-3} \mathrm{~mol}\right)$ & 38.9 & 37.6 & 38.6 & 38.6 & 22.7 & 21.7 & 22.4 & 22.5 \\
\hline$M_{t o t} / M_{R a z, i n j}$ & $68.3 \%$ & $66.0 \%$ & $67.7 \%$ & $67.7 \%$ & $39.9 \%$ & $38.1 \%$ & $39.4 \%$ & $39.4 \%$ \\
\hline
\end{tabular}


(a) Section St1

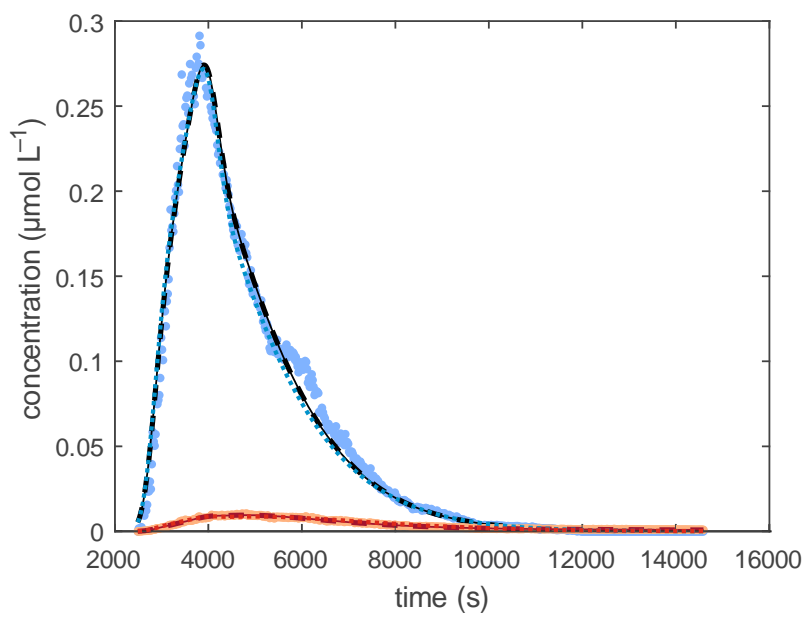

(b) Section St1

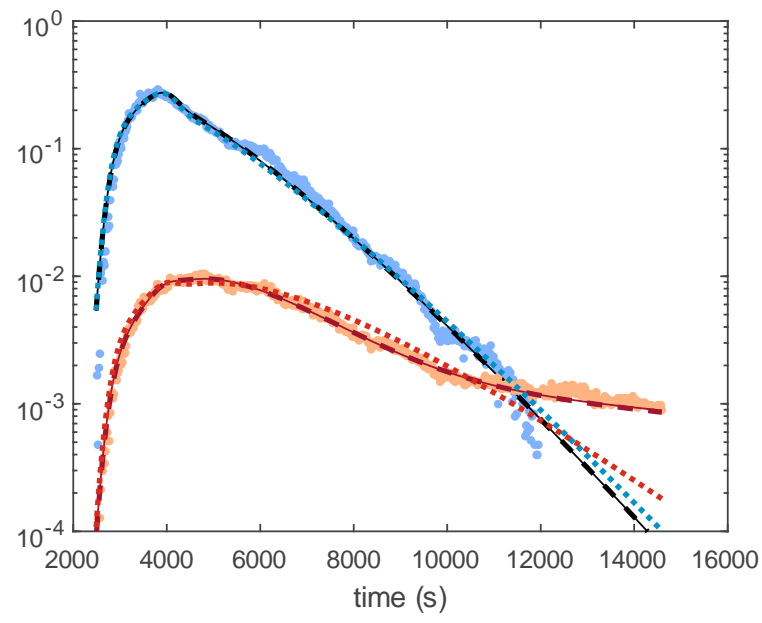

\begin{tabular}{|c|c|c|}
\hline $\begin{array}{c}\text { Raz, measured } \\
\text { - Raz, 2SZ-S } \\
\text { - - - Raz, 2SZ-P }\end{array}$ & $\begin{array}{c}\text { Rru, measured } \\
\text { - Rru, 2SZ-S } \\
\text { - - - Rru, 2SZ-P }\end{array}$ & R Raz, 1SZ \\
\hline
\end{tabular}

(c) Section St2

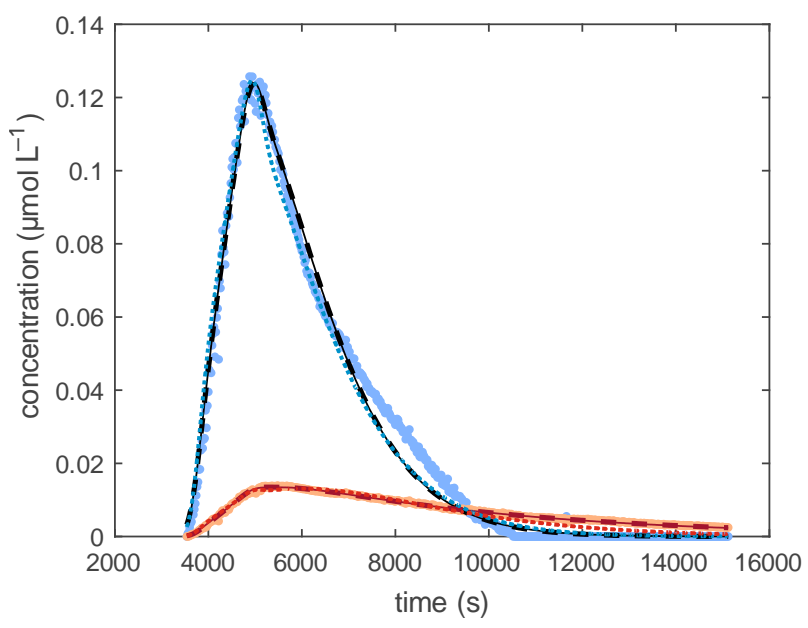

(d) Section St2

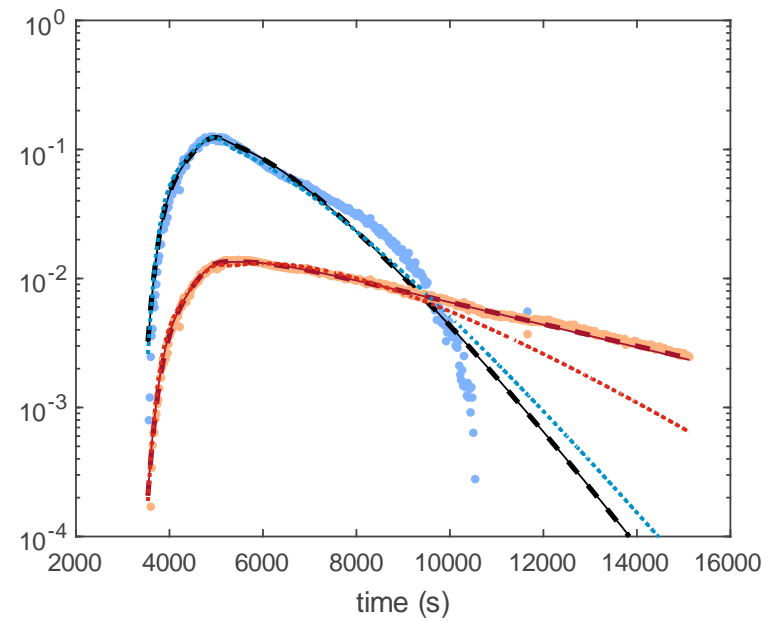

Figure 4 Observed and best-fit BTCs for in-series (solid lines) and in-parallel (dashed lines) arrangements, for both Raz and Rru. The curves are shown in linear and logarithmic scales (left and right panels, respectively), for section St1 in the first row and section St2 in the second row.

\subsubsection{Calibration Results}

The best-fit values of the calibrated model parameters are reported in Table 3. Dispersion coefficients have similar values, i.e., 0.016 and $0.014-0.015 \mathrm{~m}^{2} \mathrm{~s}^{-1}$ for the first and second reach, respectively. Both the 2SZ-P and 2SZ-S models conceptually assume two storage zones, the first (SZ1) associated with fast storage, and the second (SZ2) with slower storage. As shown in Table 3, exchange rates $\alpha_{i}$ and mean residence times $T_{i}$, where $i=1,2$, have similar orders of magnitude in both reaches. For both the 2SZ-P and 2SZ-S models, the transfer rates $\alpha_{1}$ are significantly different from the transfer rates $\alpha_{2}$. This is more evident for the parallel arrangement where $\alpha_{1}$ and $\alpha_{2}$ differ approximately by an order of magnitude in both reaches. Similar differences are found for $T_{1}$ and $T_{2}$ differ by almost an order of magnitude at both 
sections and for both arrangements, and the higher values of $T_{2}$ can be associated with a significant second transient storage component. This is more evident for the first reach R01, where $\frac{T_{2}}{T_{1}}=28-80$, and where the tail of the Resorufin BTC exhibits a change of slope in a semi-log plot (Figure $4 \mathrm{~b}$ ). It must be noted that the uncertainty in the mean residence time $T_{2}$ for reach R01 is relatively high, as the tail of the Rru BTC would need to be measured over a longer time period to determine this parameter with a narrower confidence interval. For reach R02 the ratio $\frac{T_{2}}{T_{1}}=4.9-6.8$ indicates that the two storage zones are still distinguishable, but with a smaller difference between the mean residence times than that found in reach R01, and a significantly smaller uncertainty in the parameter $T_{2}$. Hyporheic flows, typically associated with high $T_{2}$, could have been partially inhibited by bottom vegetation covering the second part of reach R02, corresponding to reach R12.

With reference to the transformation rates of the smart tracer (Table 3), it can be observed that for both reaches and for both arrangements of the storage zones, $\lambda_{12}$ is generally higher in the SZ1 than in the MFC, and at least an order of magnitude higher in SZ1 compared to SZ2 $\left(\lambda_{W 12}<\lambda_{S 12,1}<\lambda_{S 12,2}\right)$. This implies that the conversion of Raz to Rru mostly occurs in the storage zones, and in particular in the slow retention zone. All decay rates $\lambda_{W 12}, \lambda_{S 12,1}$ and $\lambda_{S 12,2}$ increase by at least an order of magnitude from section $\mathrm{St} 1$ to section $\mathrm{St} 2$, probably due to the fact that the final part of R02, corresponding to reach R12, is highly vegetated and with a less regular bed than reach R01, leading to a higher biological activity both in the main flow channel and in the two retention zones. However, the uncertainty in the transformation rates in the second storage zone, $\lambda_{S 12,2}$, is high, particularly for the model with the two storage zones in parallel.

For Reach R01, the Raz decay rates $\lambda_{W 1}(\mathrm{MFC})$ and $\lambda_{S 1,1}(\mathrm{SZ1})$ are very small under both arrangements of the storage zones, whereas the decay rate $\lambda_{S 1,2}(\mathrm{SZ2})$ can be several orders of magnitude higher than $\lambda_{W 1}$ and $\lambda_{S 1,1}$. The decay rates for Rru, $\lambda_{W 2}$ (MFC), $\lambda_{S 2,1}$ (SZ1) and $\lambda_{S 2,2}$ (SZ2), are relatively small, and have higher values in SZ1 in the case of the two-storage zone model. However, except for $\lambda_{S 1,1}$ in the $1 \mathrm{SZ}$ model, and $\lambda_{S 2,1}$ and $\lambda_{S 1,2}$ in the 2SZ-P and 2SZ-S models, the confidence intervals indicate that the decay rates for both Raz and Rru are generally small enough to not significantly affect the quality of the fits. 
Table 3. Calibrated parameters for the single-storage zone model (1SZ) and the two-storage zone model with zones arranged in series (2SZ-S) and in parallel (2SZ-P). The values between parentheses represent 95\% confidence intervals.

\begin{tabular}{|c|c|c|c|c|c|c|}
\hline Section & St1 & St1 & St1 & St2 & St2 & St2 \\
\hline Model & $1 \mathrm{SZ}$ & $2 \mathrm{SZ}-\mathrm{S}$ & 2SZ-P & $1 \mathrm{SZ}$ & $2 \mathrm{SZ}-\mathrm{S}$ & 2SZ-P \\
\hline$A\left(\mathrm{~m}^{2}\right)$ & 1.18 & 1.18 & 1.18 & 0.93 & 0.93 & 0.93 \\
\hline$Q\left(\mathrm{~m}^{3} \mathrm{~s}^{-1}\right)$ & 0.054 & 0.054 & 0.054 & 0.054 & 0.054 & 0.054 \\
\hline \multirow[t]{2}{*}{$D\left(\times 10^{-2} \mathrm{~m}^{2} \mathrm{~s}^{-1}\right)$} & 1.6 & 1.6 & 1.6 & 1.1 & 1.4 & 1.5 \\
\hline & $(1.0-2.1)$ & $(1.0-2.0)$ & $(1.3-1.7)$ & $(0.55-1.7)$ & $(0.78-1.9)$ & $(0.93-1.8)$ \\
\hline \multirow[t]{2}{*}{$\alpha_{1}\left(\times 10^{-4} \mathrm{~s}^{-1}\right)$} & 6.7 & 7.0 & 5.73 & 8.2 & 8.2 & 6.2 \\
\hline & $(6.5-6.9)$ & $(6.8-7.2)$ & $(5.54-6.03)$ & $(8.0-8.4)$ & $(8.0-8.7)$ & $(5.9-6.5)$ \\
\hline \multirow[t]{2}{*}{$T_{1}\left(\times 10^{3} \mathrm{~s}\right)$} & 1.1 & 1.0 & 0.82 & 1.1 & 0.84 & 0.65 \\
\hline & $(1.07-1.13)$ & $(0.97-1.02)$ & $(0.79-0.84)$ & $(1.06-1.14)$ & $(0.75-0.88)$ & $(0.61-0.73)$ \\
\hline \multirow[t]{2}{*}{$\alpha_{2}\left(\times 10^{-4} \mathrm{~s}^{-1}\right)$} & & 2.4 & 1.3 & & 3.5 & 0.96 \\
\hline & & $(2.2-2.5)$ & $(0.80-1.4)$ & & $(2.7-3.6)$ & $(0.83-1.9)$ \\
\hline \multirow[t]{2}{*}{$T_{2}\left(\times 10^{3} \mathrm{~s}\right)$} & & 28 & 66 & & 4.2 & 4.4 \\
\hline & & $(11-105)$ & $(42-123)$ & & $(3.6-5.1)$ & $(4.0-5.0)$ \\
\hline \multirow[t]{2}{*}{$\lambda_{W 1}\left(\times 10^{-4} \mathrm{~s}^{-1}\right)$} & 0 & 0.020 & 0 & 0 & 0.64 & 1.8 \\
\hline & $(0-0.056)$ & $(0-0.055)$ & $(0-0.11)$ & $(0-0.072)$ & $(0.33-0.72)$ & $(1.2-2.0)$ \\
\hline \multirow[t]{2}{*}{$\lambda_{W 12}\left(\times 10^{-4} \mathrm{~s}^{-1}\right)$} & 0.080 & 0.068 & 0.083 & 0.20 & 0.18 & 0.12 \\
\hline & $(0.074-0.085)$ & $(0.064-0.073)$ & $(0.065-0.089)$ & $(0.19-0.21)$ & $(0.17-0.22)$ & $(0.11-0.14)$ \\
\hline \multirow[t]{2}{*}{$\lambda_{W 2}\left(\times 10^{-4} \mathrm{~s}^{-1}\right)$} & 0 & 0.001 & 1.65 & 0 & 0 & 0.005 \\
\hline & $(0-0.28)$ & $(0-0.64)$ & $(0.17-2.3)$ & $(0-0.44)$ & $(0-0.080)$ & $(0-0.17)$ \\
\hline \multirow[t]{2}{*}{$\lambda_{S 1,1}\left(\times 10^{-4} \mathrm{~s}^{-1}\right)$} & 2.7 & 0.027 & 0.078 & 4.7 & 0 & 0.019 \\
\hline & $(2.5-2.8)$ & $(0-0.43)$ & $(0-0.13)$ & $(4.5-4.9)$ & $(0-0.083)$ & $(0-0.30)$ \\
\hline \multirow[t]{2}{*}{$\lambda_{S 12,1}\left(\times 10^{-4} \mathrm{~s}^{-1}\right)$} & 0.20 & 0.35 & 0.42 & 0.20 & 1.2 & 0.44 \\
\hline & $(0.19-0.22)$ & $(0.32-0.41)$ & $(0.35-0.45)$ & $(0.18-0.23)$ & $(1.1-1.4)$ & $(0.31-0.82)$ \\
\hline \multirow[t]{2}{*}{$\lambda_{S 2,1}\left(\times 10^{-4} \mathrm{~s}^{-1}\right)$} & 0 & 1.6 & 1.77 & 0 & 18 & 35 \\
\hline & $(0-0.13)$ & $(1.5-2.4)$ & $(1.4-2.0)$ & $(0-0.076)$ & $(16-21)$ & $(20-51)$ \\
\hline \multirow[t]{2}{*}{$\lambda_{S 1,2}\left(\times 10^{-4} \mathrm{~s}^{-1}\right)$} & & 260 & 43 & & 0 & 58 \\
\hline & & $(62-390)$ & $(15-981)$ & & $(0-0.20)$ & $(8-610)$ \\
\hline \multirow[t]{2}{*}{$\lambda_{S 12,2}\left(\times 10^{-4} \mathrm{~s}^{-1}\right)$} & & 48 & 28 & & 25 & 240 \\
\hline & & $(4.3-425)$ & $(5.1-881)$ & & $(21-44)$ & (77-990) \\
\hline \multirow[t]{2}{*}{$\lambda_{S 2,2}\left(\times 10^{-4} \mathrm{~s}^{-1}\right)$} & & 0 & 0.030 & & 0 & 0.008 \\
\hline & & $(0-0.13)$ & $(0-0.040)$ & & $(0-0.82)$ & $(0-0.18)$ \\
\hline$\epsilon_{m}$ & 0.122 & 0.0731 & 0.0732 & 0.129 & 0.0966 & 0.0963 \\
\hline
\end{tabular}


The uncertainty of the model parameters was further analysed by sampling each of the parameters within broad intervals and calibrating the remaining parameters. The parallel axis plot in Figure 5 provides a visual representation of the resulting sets of parameter values and the corresponding fitting errors expressed as a percentage deviation from the overall minimum

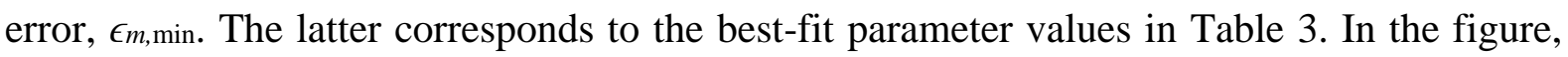
best fits with errors deviating by less than $5 \%$ from the overall minimum, $\epsilon_{m}$, min, are highlighted with a red shade, with varying intensity depending on the deviation. A similar plot is provided for section St1 in the supplementary material (Figure S1).

For the two storage-zone model, it can be seen that the decay rates $\lambda_{W 1}, \lambda_{W 2}, \lambda_{S 1,1}, \lambda_{S 2,1}$, $\lambda_{S 1,2}, \lambda_{S 2,2}$ and to a minor extent $\lambda_{S 2,1}$, can be varied within a broad range with little change in the error, whereas the transformation rates $\lambda_{W 12}$ and $\lambda_{S 12,1}$ are reasonably well-determined. The uncertainty of $\lambda_{W 12,2}$ is lower than that of the individual decay rates, but relatively high compared to that of $\lambda_{W 12}$ and $\lambda_{S 12,1}$. Even the more parsimonious $1 \mathrm{SZ}$ model is not particularly sensitive to the decay rates when these are smaller than a certain threshold $\left(\sim 10^{-5} \mathrm{~s}^{-1}\right)$. This suggests that the decay rates should be either imposed, if these are known from laboratory tests, or simply set to zero if they are found to be unacceptably uncertain. On the other hand, the relatively high sensitivity of both the $1 \mathrm{SZ}$ and $2 \mathrm{SZ}$ models to the transformation rates $\lambda_{W 12}$ and $\lambda_{S 12,1}$ appears to support the assumption that stream metabolic rates are identifiable from smart tracer data, but further information may be needed if the transformation rate $\lambda_{W 12,2}$ for the second storage zone is to be determined with an acceptable level of confidence. As other studies have suggested (Lemke et al., 2013; Kelleher et al., 2019), the uncertainty in the transport parameters may be further reduced if tracer tests are conducted using a conservative tracer in addition to Resazurin. It should also be pointed out that the general residence time formulation of STIR-RST allows storage processes to be potentially represented by specific physically based models, which may in turn be a function of system parameters that can be directly measured or estimated. 
(a) Single storage zone, $\epsilon_{m, \min }=0.129$

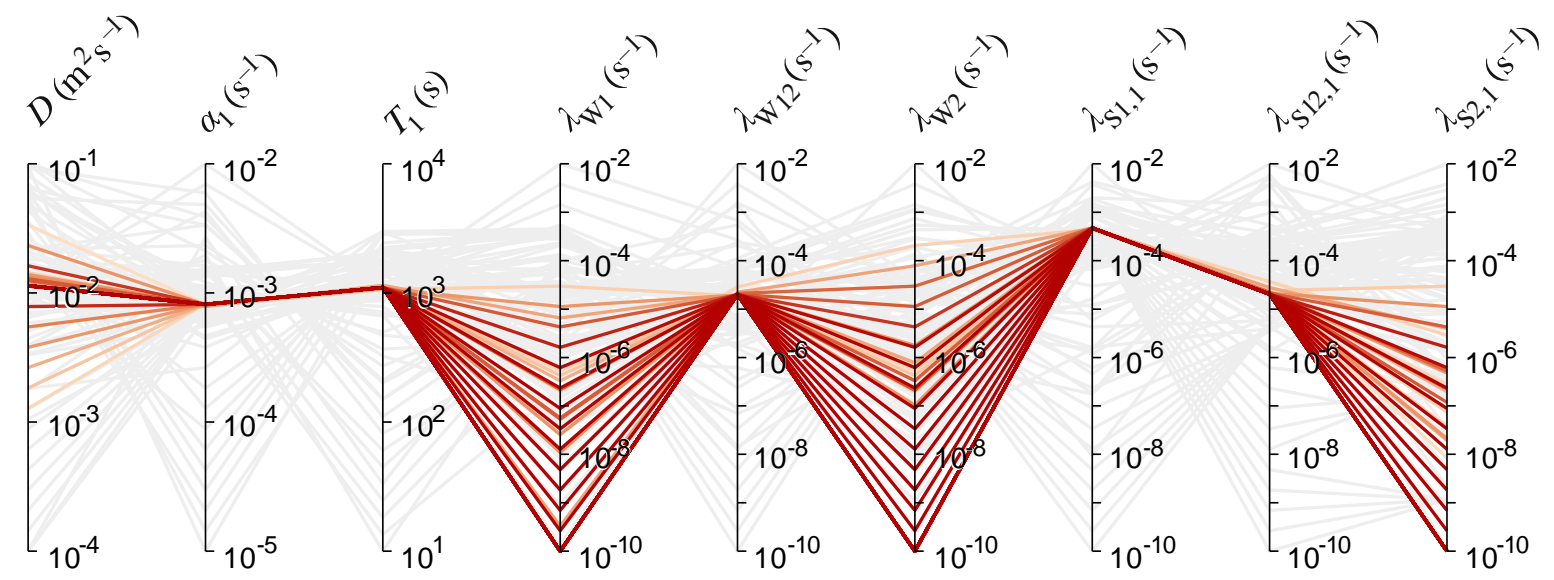

(b) Two storage zones in parallel, $\epsilon_{m, \min }=0.0963$

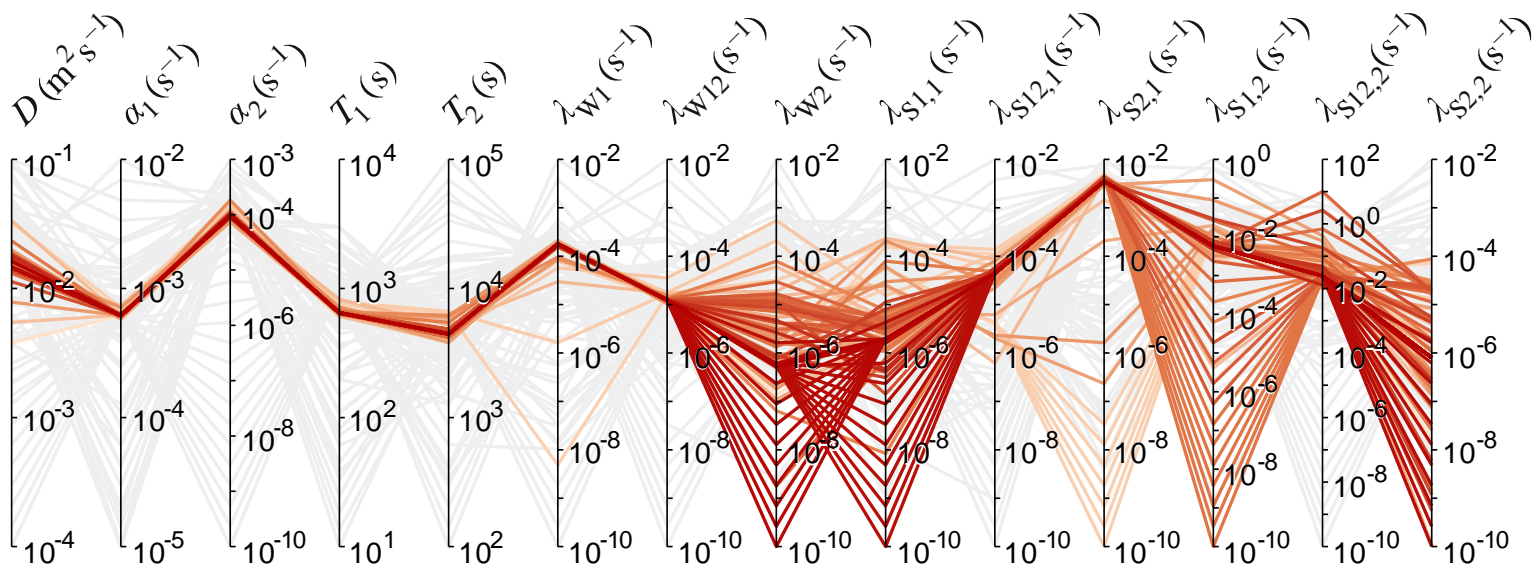

(c) Two storage zones in series, $\epsilon_{m, \min }=0.0966$
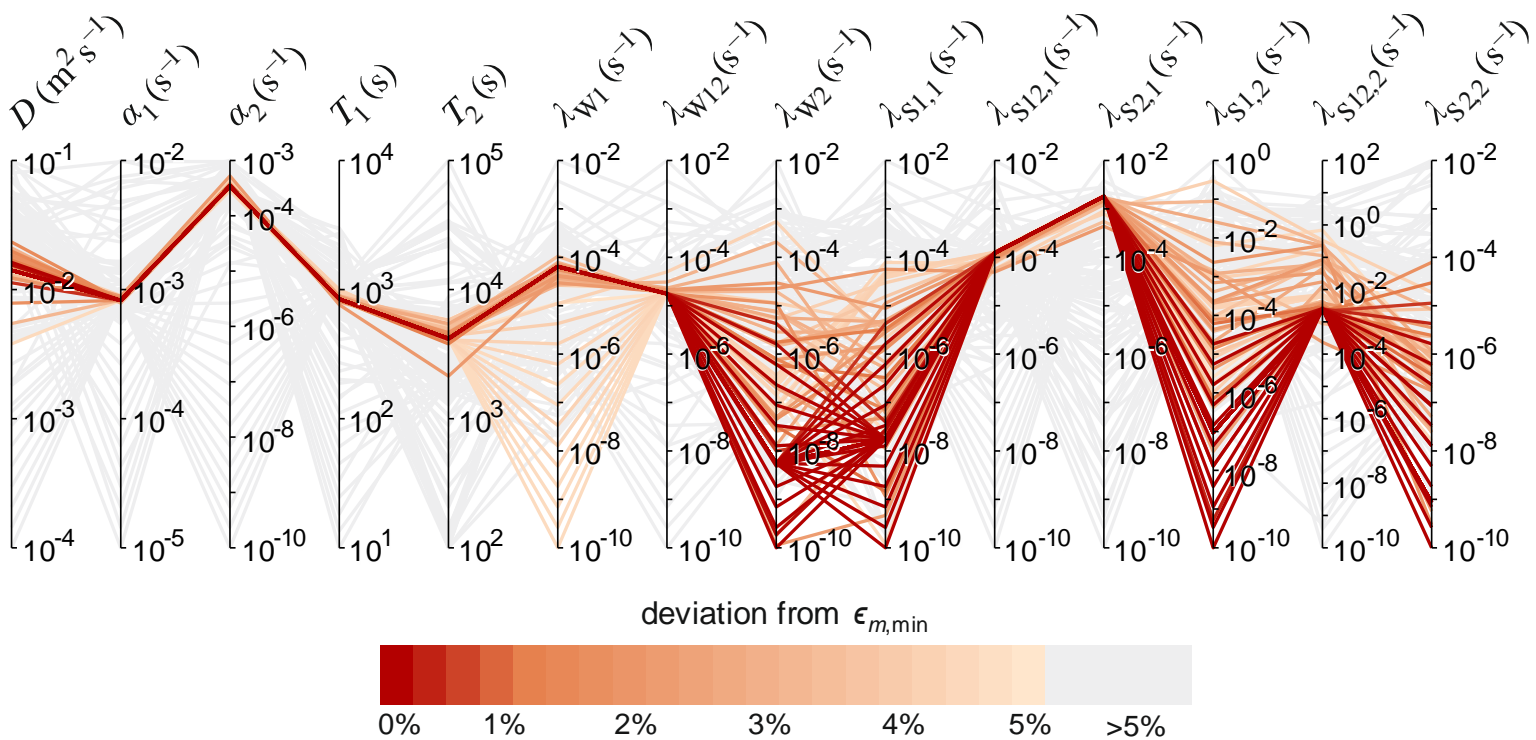

Figure 5. Parallel axis plot representing sets of best-fit parameter values for the BTCs at section St2, obtained by imposing one of the parameters and optimizing the others. The line color represents the deviation from the global best-fit, quantified as a percentage deviation from the minimum error, $\epsilon_{m, \min }$. 


\section{Conclusions}

One-dimensional transport models are widely used in water quality models to predict concentrations of nutrients and pollutants in stream corridors. Model parameters are often calibrated using experimental data from field tracer studies. Recent advancements in tracer techniques have seen the development of smart tracer techniques that can provide information about microbiological activity and sediment-water interactions. In the present study, a general residence time formulation was presented for modelling transport and reaction processes in stream channels. The stochastic formulation of the STIR model (Marion et al, 2008) was extended to represent reaction and transformation of Raz-Rru smart tracers.

The applicability of the proposed modelling framework to Raz-Rru tracer data from field experiments was demonstrated assuming transient storage with two distinct storage zones, each characterized by an exponential RTD, with two possible arrangements of the storage zones: nested (in-series) or competing (in-parallel). The calibrated model was found to well reproduce the observed BTCs, and the values of the estimated parameters appeared to well match the physical features of the two reaches and the expected biochemical characteristics. If only one storage zone is considered, the model still well fits the bulk of the BTCs but fails to reproduce the tails of the curves. Although in this particular application, good fits and physically reasonable parameters were obtained under both assumptions of in-parallel and in-series arrangement of the storage zones, the possibility of having two different conceptual arrangements of the storage compartments allows the modeler to evaluate the sensitivity of the calibrated parameters to different storage assumptions. In order to reduce the uncertainty in the model parameters, discharge and flow cross-sectional area must generally be determined from direct measurements. The use of a conservative tracer in addition to a smart tracer can also reduce uncertainty by providing an estimate of the transport and storage parameters independently of the reaction rates.

The STIR-RST toolbox provides an object-oriented framework in which new transport and storage models can be developed as subclasses of a base model class. The toolbox also provides a user-friendly command interface for managing input/output data and model settings, and allows parameter calibration using state-of-the-art methods for global and local optimization. This makes STIR-RST a flexible tool for testing and evaluating alternative models of reactive transport and transient storage, and for characterising stream transport processes using tracer data. 


\section{Acknowledgments}

The authors thank Dr. Giovanni Marco Carrer (LASA Environmental System Analysis Lab, University of Padova) for his scientific and technical support in laboratory and field activities. A special thanks also goes to Julia Knapp and two anonymous reviewers for their constructive comments and useful criticism.

\section{References}

Argerich, A., Haggerty, R., Marti, E., Sabater, F., Zarnetske, J. (2011), Quantification of metabolically active transient storage (MATS) in two reaches with contrasting transient storage and ecosystem respiration. Journal of Geophysical Research, 116, G03034, doi:10.1029/2010JG001379.

Beer, T., Young, P. C. (1983), Longitudinal Dispersion in Natural Streams. Journal of Environmental Engineering, 109, 1049-1067. doi:10.1061/(ASCE)07339372(1983)109:5(1049).

Bencala, K. E., Walters, R. A. (1983), Simulation of solute transport in a mountain pool-andriffle stream-A transient storage model, Water Resources Research, 19(3), 718-724, doi:10.1029/WR019i003p00718.

Boano, F., A. I. Packman, A. Cortis, R. Revelli, and L. Ridolfi (2007b), A continuous time random walk approach to the stream transport of solutes, Water Resources Research, 33, W10425, doi:10.1029/2007WR006062.

Bottacin-Busolin, A., A. Marion, T. Musner, M. Tregnaghi, and M. Zaramella (2011), Evidence of distinct contaminant transport patterns in rivers using tracer tests and a multiple domain retention model, Advances in Water Resources, 34, 737- 746.

Bottacin-Busolin, A. (2019), Modeling the effect of hyporheic mixing on stream solute transport, Water Resources Research, 55, 9995-10011, doi:10.1029/2019WR025697.

Briggs, M. A., M. N. Gooseff, C. D. Arp, and M. A. Baker (2009), A method for estimating surface transient storage parameters for streams with concurrent hyporheic storage, Water Resources Research, 45, W00D27, doi:10.1029/2008WR006959

Choi, J., J. W. Harvey, and M. H. Conklin (2000), Characterizing multiple timescales of stream and storage zone interaction that affect solute fate and transport in streams, Water Resources Research, 36, 1511- 1518, doi:10.1029/2000WR900051.

Deng, Z.-Q., L. Bengtsson, and V. P. Singh (2006), Parameter estimation for fractional dispersion model for rivers, Environ. Fluid. Mech., 6(5), 451- 475.

Elliott, A. H., \& Brooks, N. H. (1997), Transfer of nonsorbing solutes to a streambed with bed forms: Theory. Water Resources Research, 33( 1), 123-136, doi:10.1029/96WR02784.

Fischer, H. B., List, J. E., Koh, C. R., Imberger, J., \& Brooks, N. H. (1979), Mixing in Inland and Coastal Waters, Academic Press.

González-Pinzón, R., Haggerty, R., \& Argerich, A. (2014), Quantifying spatial differences in metabolism in headwater streams. Freshwater Science, 33(3), 798-811, doi: $10.1086 / 677555$. 
González-Pinzón, R., Ward, A. S., Hatch, C. E., Wlostowski, A. N., Singha, K., Gooseff, M. N., et al. (2015), A field comparison of multiple techniques to quantify groundwatersurface-water interactions. Freshwater Science, 34(1), 139-160, doi:10.1086/679738.

González-Pinzón, R., Peipoch, M., Haggerty, R., Martí, E., \& Fleckenstein, J.H. (2016), Night time and day time respiration in a head water stream. Ecohydrology, 9(1), 93100, doi:10.1002/eco.1615.

Gooseff, M. N., McKnight, D. M., Runkel, R. L., \& Duff, J. H. (2004), Denitrification and hydrologic transient storage in a glacial melt water stream, McMurdo Dry Valleys, Antarctica. Limnology and Oceanography, 49(5), 1884- 1895, doi:10.4319/lo.2004.49.5.1884.

Haario, H., Laine, M., Mira, A., and Saksman, E. (2006), DRAM: Efficient adaptive MCMC, Statistics and Computing, 16(4), 339-354. doi: 10.1007/s11222-006-9438-0.

Haggerty, R., A. Argerich, and E. Martí (2008), Development of a "smart" tracer for the assessment of microbiological activity and sediment-water interaction in natural waters: The Resazurin-Resorufin system, Water Resources Research, 44, W00D01, doi:10.1029/2007WR006670.

Haggerty, R., and P. C. Reeves (2002), STAMMT-L 1.0, formulation and user's guide, Tech. Rep. ERMS \#520308, Sandia Natl. Lab., Albuquerque, N. M.

Kelly, J. F., Bolster, D., Meerschaert, M. M., Drummond, J. D., and Packman, A. I. (2017), FracFit: A robust parameter estimation tool for fractional calculus models, Water Resources Research , 53, 2559-2567, doi:10.1002/2016WR019748.

Kelleher, C., Ward, A., Knapp, J. L. A., Blaen, P. J., Kurz, M. J., Drummond, J. D., et al. (2019). Exploring Tracer Information and Model Framework Trade-Offs to Improve Estimation of Stream Transient Storage Processes. Water Resources Research, 55(4), 3481-3501. https://doi.org/10.1029/2018WR023585.

Kerr, P.C. ,Gooseff, M.N., Bolster, D. (2013), The significance of model structure in onedimensional stream solute transport models with multiple transient storage zones competing vs. nested arrangements, Journal of Hydrology, Volume 497, 2013, Pages 133-144, doi:10.1016/j.jhydrol.2013.05.013.

Knapp, J. L. A., Cirpka, O. A. (2017). Determination of hyporheic travel time distributions and other parameters from concurrent conservative and reactive tracer tests by localin-global optimization. Water Resources Research, 53(6), 4984-5001, https://doi.org/10.1002/2017WR020734.

Knapp, J. L. A., González-Pinzón, R., Haggerty, R. (2018), The resazurin-resorufin system: Insights from a decade of "smart" tracer development for hydrologic applications. Water Resources Research ,54, 6877-6889, doi:10.1029/2018WR023103.

Lemke, D., Liao, Z. J., Wohling, T., Osenbruck, K., Cirpka, O. A. (2013), Concurrent conservative and reactive tracer tests in a stream undergoing hyporheic exchange. Water Resources Research, 49, 3024-3037, doi:10.1002/wrcr.20277.

Liao, Z., Cirpka, O. A. (2011). Shape-free inference of hyporheic traveltime distributions from synthetic conservative and "smart" tracer tests in streams. Water Resources Research, 47(7). https://doi.org/10.1029/2010WR009927. 
Liao, Z., Lemke, D., Osenbrück, K., Cirpka, O. A. (2013). Modeling and inverting reactive stream tracers undergoing two-site sorption and decay in the hyporheic zone. Water Resources Research, 49(6), 3406-3422. https://doi.org/10.1002/wrcr.20276.

Marion, A., M. Zaramella, and A. Bottacin-Busolin (2008b), Solute transport in rivers with multiple storage zones: The STIR model, Water Resources Research 44, W10406, doi:10.1029/2008WR007037.

Rana, S.M.M., Boccelli. D.L., Scott, D.T., Hester, E.T. (2019), Parameter uncertainty with flow variation of the one-dimensional solute transport model for small streams using Markov chain Monte Carlo, Journal of Hydrology, 575 (2019), 1145-1154, 10.1016/j.jhydrol.2019.06.003.

Runkel, R. L. (1998), One-dimensional transport with inflow and storage (OTIS): A solute transport model for streams and rivers, edited, US Department of the Interior, US Geological Survey.

Runkel, R. L., and S. C. Chapra(1993), An efficient numerical solution of the transient storage equations for solute transport in small streams, Water Resources Research, 29, 211-215, doi:10.1029/92WR02217.

Smith, P., Beven, K., Tawn, J., Blazkova, S., Merta, L. (2006), Discharge-dependent pollutant dispersion in rivers: Estimation of aggregated dead zone parameters with surrogate data, Water Resources Research, 42, doi:10.1029/2005WR004008.

Yakirevich, A., Shelton, D., Hill, R., Kiefer, L., Stocker, M., Blaustein, R., Kuznetsov, M., McCarty, G., Pachepsky, Y. (2017), Transport of Conservative and "Smart" Tracers in a First-Order Creek: Role of Transient Storage Type. Water 2017, 9, 485.

Young, P. C., Garnier, H. (2006). Identification and estimation of continuous-time, databased mechanistic (DBM) models for environmental systems. Environmental Modelling \& Software, 21(8), 1055-1072, doi:10.1016/j.envsoft.2005.05.007.

Wallis, S., Young, P., Beven, K. (1989), Experimental investigation of the aggregated dead zone model, Proceedings of the Institution of Civil Engineers 87, 1-22, doi:10.1680/iicep.1989.1450.

Wörman, A., A. I. Packman, and K. Jonsson (2002), Effect of flow-induced exchange in hyporheic zones on longitudinal transport of solutes in stream and rivers, Water Resources Research, 38(1), 1001, doi:10.1029/2001WR000769.

Zaramella, M., Marion, A., Lewandowski, J., Nützmann, G. (2016), Assessment of transient storage exchange and advection-dispersion mechanisms from concentration signatures along breakthrough curves. Journal of Hydrology, 538, 794- 801. 